\title{
Primary HIV-1 Infection: Diagnosis, Pathogenesis, and Treatment
}

\author{
Malini Soogoor, $M D$, and Eric S. Daar, $M D^{*}$
}

\author{
Address \\ *Division of HIV Medicine, Los Angeles BioMedical Research Institute \\ at Harbor-UCLA Medical Center; David Geffen School of Medicine at \\ UCLA, II 24 West Carson Street, N-24, Torrance, CA 90502, USA. \\ E-mail: edaar@labiomed.org \\ Current Infectious Disease Reports 2005, 7:147-153 \\ Current Science Inc. ISSN I523-3847 \\ Copyright () 2005 by Current Science Inc.
}

Primary HIV-I infection represents the time when the virus is first disseminating throughout the body and induces host immune responses. Diagnosing this stage of disease requires an understanding of who is at risk, the clinical manifestations of primary infection, and how the diagnosis is made. Identifying these individuals allows for counseling to prevent further transmission to others and the potential benefits associated with early antiretroviral therapy. Moreover, studying these individuals provides important insight into the biology of HIV-I transmission and immunopathogenesis.

\section{Introduction}

As of 2003, the Joint United Nations Program on AIDS reported approximately 40 million people living with HIV-1 infection worldwide. In the United States, it is estimated that the number of infected persons is approaching 1 million, with the number of annual incident cases remaining stable at approximately 40,000 cases per year. Moreover, it is estimated that as many as $25 \%$ of these individuals are unaware of their HIV-1 status. Attempts to control this epidemic include enhancing the availability of and access to testing, and providing education and resources in order to facilitate prevention while awaiting the development of effective microbicides and vaccines.

Primary HIV-1 infection occurs shortly after exposure with widespread viremia and the initiation of host immune responses. Identifying these patients provides a unique opportunity to better understand the psychosocial factors associated with persons continuing to place themselves at risk for infection. In addition, this is a highly dynamic stage of disease at which time host-virus interactions are occurring that predict long-term outcomes. Most importantly, identification of primary HIV-1 infection allows for early diagnosis, counseling, and entry into care for those actively putting themselves and their partners at risk for infection. This review summarizes issues surrounding the identification and diagnosis of primary HIV-1 infection, what has been learned about immunopathogenesis, and the potential role of early antiretroviral therapy in this setting.

Primary HIV-1 Infection: Definition and Diagnosis

Primary or acute HIV-1 infection is characterized by high levels of plasma HIV-1 RNA and is often associated with an "acute retroviral syndrome" that usually includes fever with a variety of nonspecific clinical and laboratory abnormalities. The time from exposure to symptomatic disease is typically 2 to 4 weeks, and the duration of illness is generally days to weeks. Identifying patients with this syndrome requires a thorough risk assessment, recognition of the variable clinical and laboratory manifestations, and understanding what tests need to be performed in order to make the diagnosis.

\section{Defining primary HIV-1 infection}

The definition of primary HIV-1 infection varies throughout the literature. Some studies include patients with evolving humoral immune responses, such as negative, indeterminate, or partially positive Western blots, whereas others include anyone with documentation of being HIV-1-antibody negative in the preceding 6 or 12 months. The definition used influences the methods needed to make the diagnosis and any considerations regarding the pathogenic implications of this stage of disease. Consequently, when reviewing studies of primary HIV-1 infection, it is necessary to assess the patient population included in the analyses.

\section{Clinical manifestations of primary HIV-1 infection}

Primary HIV-1 infection is classically described as a "mononucleosis-like" illness with fever, sore throat, and cervical adenopathy. Nevertheless, the presentation can be highly variable, with some being asymptomatic, others experiencing a mild nonspecific illness that may not result in seeking medical attention, and those who develop a severe illness with considerable morbidity 
Table I. Signs and symptoms in at-risk individuals referred for screening who were or were not diagnosed with PHI

\begin{tabular}{|c|c|c|c|}
\hline Signs/symptoms & PHI*, \% $(n=218)$ & $\mathrm{PHI}^{\dagger}, \%(n=40)$ & Non-PHIt, \% $(n=164)$ \\
\hline Fever & 77 & 88 & 50 \\
\hline Myalgia & 54 & 60 & 26 \\
\hline Skin rash & 56 & 58 & 21 \\
\hline Night sweats & 22 & 50 & 32 \\
\hline Lethargy/malaise & 66 & 72 & 58 \\
\hline Arthralgia & 31 & 28 & 13 \\
\hline Sore throat & 45 & 42 & 49 \\
\hline Cervical adenopathy & 39 & 38 & 29 \\
\hline Oral ulcers & 29 & 8 & 8 \\
\hline Headache & 51 & 55 & 44 \\
\hline Thrush & 17 & 5 & 4 \\
\hline Cough/nasal congestion & 22 & 18 & 38 \\
\hline
\end{tabular}

$[1,2]$. The most common signs and symptoms reported in the literature are included in Table 1. Common laboratory abnormalities include anemia, leukopenia, thrombocytopenia, and mild transaminase elevation. Based on the physical presentation and laboratory abnormalities, the differential diagnosis can be quite broad (Table 2). Although several groups have suggested that the severity of the acute retroviral syndrome may predict the rapidity of disease progression [3], data are somewhat limited by the many difficult to control for confounders.

Because the presenting signs and symptoms are nonspecific, several groups have attempted to define whether any single or combination of manifestations are sufficiently sensitive and specific to justify targeted screening $[2,4]$. We previously reported that those referred for screening who were diagnosed with primary infection were more likely to have fever, myalgia, arthralgia, night sweats, and rash than were those who were found not to have primary infection (Table 1); however, no single symptom or constellation of symptoms had sufficient positive or negative predictive value to allow for more targeted screening [2]. Consequently, any person presenting with an unexplained acute illness who has potentially been exposed to HIV-1 should be evaluated for primary infection.

\section{Diagnosing primary HIV-1 infection}

The first step in diagnosing this stage of disease is recognizing who is at risk. Unprotected sexual intercourse and needle sharing remain the major risk factors for HIV-1 transmission among adolescents and adults. Other confounders clearly contribute to the overall risk, such as the type of exposure, the presence of sexually transmitted infections, history of incarceration, depression, feeling of exclusion from peer groups, drug use, trading sex for drugs or money, and even the use mediations for erectile dysfunction $[5,6]$. Transmission can also occur as a result of exposures generally thought to be of lower risk, such as insertive intercourse and oral sex [7]. Because all newly infected individuals have recently put themselves and others at risk for HIV-1 transmission, it is vital that they be diagnosed and provided counseling as early as possible. These individuals are likely to have high levels of HIV-1 circulating in blood and genital secretions, with several studies suggesting that those with primary infection may be substantially contributing to the ongoing epidemic of new infections $\left[\mathbf{8}^{\bullet}\right]$. Despite the importance of identifying primary HIV-1 infection and the attention given to educating providers to recognize this stage of disease, reports continue to show that this diagnosis is often missed [9].

Detailed studies have examined the time course from exposure to the development of various virologic and immunologic markers of infection. In one study, Fiebig et al. [10••] analyzed serial specimens from those known to experience seroconversion. These investigators showed that the approximate time from exposure to HIV-1 to detectable plasma HIV-1 RNA was 12 days, to p24 antigenemia was 17 days, and to first detectable antibodies was 22 days (Fig. 1). Several groups have attempted to define the utility of these markers in patients presenting with symptoms consistent with primary infection. Although plasma HIV-1 RNA remains a very sensitive marker, the sensitivity of p24 antigen varies by study, largely defined by the serostatus of the patients at the time of testing $[2,4]$. Because p24 antigen is rapidly complexed by antibodies, it is most useful in those who are antibody-negative, the setting in which virologic tests are most valuable. In 
Table 2. Differential diagnoses for primary HIV-I infection based

on clinical/laboratory manifestations

Differential diagnosis (select examples)

Mononucleosis-like illness

EBV, CMV, toxoplasmosis, streptococcal disease

Oral or genital ulcer disease

HSV, coxsackie virus, Behcet's syndrome, aphthous stomatitis

Rash

Syphilis, enteroviruses, rubella, rickettsial diseases, roseola, parvovirus BI9, allergic reaction, rheumatologic diseases

Meningitis/encephalitis

Enteroviruses, HSV, West Nile

Transaminase elevation

Hepatitis A, B, or C, CMV, EBV, drug reaction

CMV—cytomegalovirus; EBV—Epstein-Barr virus; HSV—herpes simplex virus.

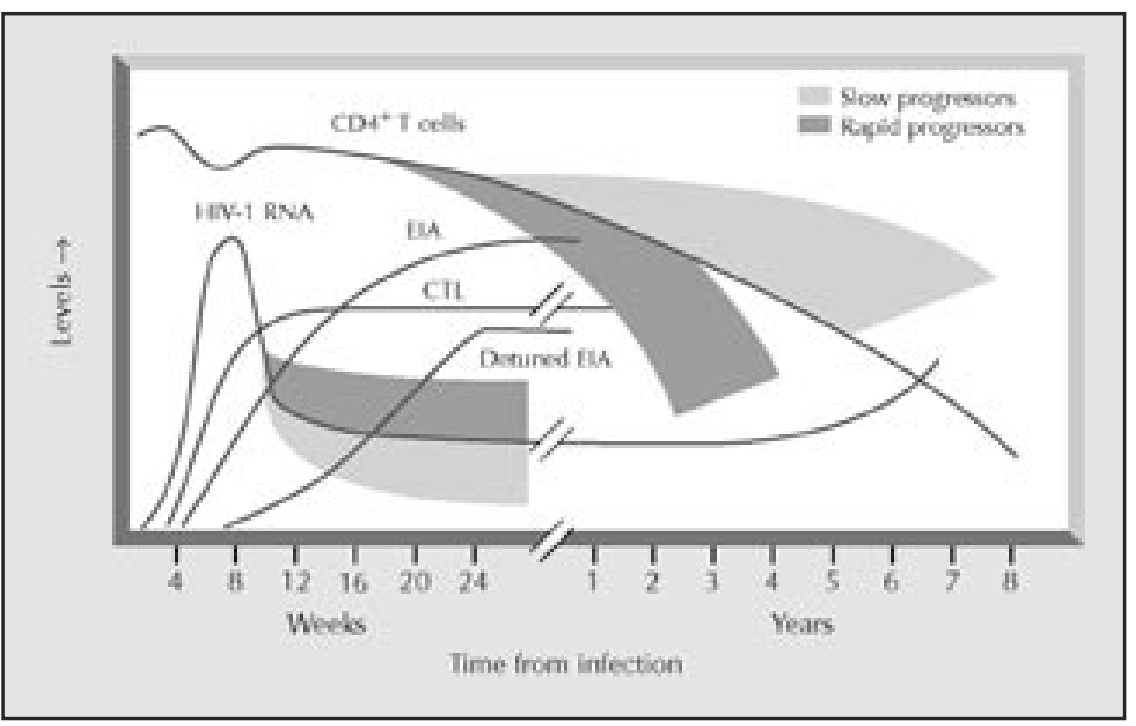

Figure I. Dynamics of virologic and immunologic events during the course of acute and chronic HIV-1 infection. CTL-cytotoxic T lymphocytes; ElA—enzyme immunoassay.

contrast, plasma HIV-1 RNA is very sensitive during all stages of primary infection, but false-positive results do occur, although levels more than 5000 copies per $\mathrm{mL}$ are rarely reported $[2,4]$. Because those with antibodynegative symptomatic primary infection have plasma HIV-1 RNA levels typically in excess of 100,000 copies per $\mathrm{mL}$, it is generally easy to distinguish false from true positive test results in this setting. In contrast, this assay should be used with great caution in those not suspected of having primary HIV-1 infection, a situation in which low-level viremia can be more difficult to interpret.

Consideration has been given to screening for seronegative HIV-1 infection in patients undergoing routine antibody testing. Such procedures are standard in blood donors and could be applied to HIV-1 testing centers by use of assays for HIV-1 antibodies and p24 antigen or HIV-1 RNA. Pooled specimens for HIV-1 RNA testing have been proposed as a potentially cost-effective means of screening for antibody-negative HIV-1 infection [11].
The utility of incorporating a viral test into routine antibody screening is supported by survey data reporting that many people presenting for HIV-1 testing do so with symptoms of primary HIV-1 infection [12].

Another novel test of interest is the less sensitive, or "detuned" enzyme immunoassay, developed to identify those who are seropositive but have been infected in the recent past (Fig. 1). These assays are most useful for epidemiologic investigations attempting to define incident cases of HIV-1 infection. Although these tests are investigational, if it is determined that there are clinically relevant implications of being known to have been infected for less than 4 to 6 months, they could be used with increasing frequency [13].

\section{Early Events in Immunopathogenesis}

Most HIV-1 infection around the world is initiated at a mucosal site. The first virion-host interaction is at the 
level of the dendritic cell, followed by spread to regional lymphoid tissue, the initiation of a specific immune response, and dissemination throughout the body. The initial high levels of viremia ultimately decrease to a nadir, and the infected person enters an asymptomatic phase of infection lasting from months to years before the development of symptomatic disease. The events that occur during primary infection provide insight into the immunopathogenesis of HIV-1 disease and important host responses to the invading pathogen that might be important for vaccine development.

\section{Early viral events}

HIV-1 infection is usually initiated by contact between the virus and tissue dendritic cells. DC-SIGN has been identified as an HIV-1-specific receptor that facilitates transport of virus across the mucosa to lymphoid tissue [14]. Infection typically is established with a macrophagetropic, nonsyncytium-inducing virus that uses the CCR5 chemokine receptor to enter the cell. Widespread seeding of most tissue and cellular compartments is established very early in the course of primary infection. It has been further shown that initial infection is usually with a relatively homogeneous population of virus that then evolves as a result of ongoing replication and immune pressure [15]. In contrast, there are other studies that show transmission of a mix of viruses from the quasispecies of the transmitting individual. Sagar et al. [16] demonstrated that those infected with multiple variants may actually experience more rapid disease progression.

During the past 5 years, there have been many reports from around the world of transmitted drug-resistant HIV1 , with the prevalence ranging from less than $5 \%$ to as high as $30 \%$ in select cohorts. Recent epidemiologic studies from the United States [17], with similar data seen in Europe, report approximately $10 \%$ of treatment-naïve individuals harbor drug-resistant virus. Although modeling has been performed to predict whether the incident of transmitted resistant virus will stabilize, increase, or decrease with time [18], trend data remain limited and variable [19]. Several studies have shown that, unlike the situation observed when resistant virus is selected for during the course of therapy, transmitted resistant HIV-1 persists as a predominant species in many who acquire such strains, even without the initiation of antiretroviral therapy [20]. Consequently, it is justifiable to perform resistance testing on patients who have been infected for months or years, as suggested by current guidelines $[21,22]$, and these viruses are likely to limit therapeutic response, regardless of when treatment is initiated [19].

\section{Early immunologic events}

The initial decrease in plasma HIV-1 RNA during the course of primary infection is temporally associated with the emergence of HIV-1-specific cytotoxic T lymphocytes [23]. In addition, several groups have demonstrated a relationship between disease progression and the breadth and magnitude of these responses [24], and viral rebound occurring in association with viral escape from cellular immunity [25]. The role of humoral immunity in controlling early HIV-1 replication is less clear because despite the early emergence of neutralizing antibodies, viral escape is seen very quickly, and escape has not been correlated with changes in viral control $[26,27]$. Other aspects of host immunity may also have an antiviral effect during primary HIV-1 infection, such as natural killer cells [28] and soluble factors [29]. Further support for the hypothesis that early host antiviral responses influence the natural history of disease is the observation that a steeper slope of HIV-1 RNA decline during primary infection is associated with slower disease progression [30].

Because $\mathrm{CD} 4^{+} \mathrm{T}$ lymphocytes are the primary targets of HIV-1 infection and are known to orchestrate host immunity, several studies have analyzed the relationship between HIV-1-specific CD4+ T-lymphocyte responses and the natural history of disease. Rosenberg et al. [31] first demonstrated that most chronically infected individuals have weak HIV-1-specific $\mathrm{CD}^{+}$T-lymphocyte responses. However, they found that individuals with markedly delayed clinical progression, so called "longterm nonprogressors," appear to maintain this aspect of the immune response to HIV-1. The importance of this immune marker is further supported by recent studies showing that plasma HIV-1 RNA set point is lower in those with the strongest initial HIV-1-specific CD4 ${ }^{+}$ T-lymphocyte responses [32]. Because these immune responses are weak or absent in most chronically infected people, Rosenberg et al. [33] proposed that early events may target these cells. In order to address this, they performed a pilot study in which they administered potent antiretroviral therapy to those identified with primary infection to determine whether this would preserve HIV1-specific immunity. They did find that those treated during this stage of disease generally maintained these immune responses. The next question, which has direct implications on the potential utility of treatment during primary infection, is whether preservation of these immune cells results in improved virologic, immunologic, and/or clinical outcomes.

\section{Antiretroviral Therapy during Primary HIV-1 Infection}

Since the availability of potent antiretroviral therapy, there has been increasing interest in the role of treatment during primary infection. The only well-powered, randomized controlled trial performed during this stage of disease administered zidovudine or placebo and showed short-term immunologic and clinical benefits [34]. In contrast, the role of potent antiretroviral therapy has not been tested in a controlled study and remains controversial, as described in a recent review by Smith et al. 
[35••]. Although viral eradication is not an achievable goal of early therapy, several potential benefits of treatment have been proposed, including the preservation of HIV-1-specific immune responses, decreasing the duration of the acute retroviral syndrome, reducing the initial viral set point and risk of rapid progression, limiting viral diversity, and possibly reducing the risk of transmission. As discussed in detail in the following text, most of these potential benefits from early therapy remain controversial and must be balanced against the risks of treatment, such as cost, acute and chronic toxicity (including gastrointestinal symptoms, dyslipidemia, and lipodystrophy [22,36]), the development of an acute retroviral syndrome at the time of treatment discontinuation [37], and perhaps most importantly, the risk of selecting for drug-resistant virus while on therapy or during treatment interruption(s) [21,38].

Although most of the potential benefits of early treatment have not been formally tested, it is unlikely that many would justify the potential risks of therapy. For example, although early treatment may well reduce transmissibility, this goal is probably best achieved by counseling [39]. For now, the principal rationale for therapy during primary infection remains the possibility of preserving HIV-1-specific immunity to allow for enhanced viral control off treatment. Despite the initial excitement surrounding the "Berlin Patient," an individual treated during primary infection who then contained viral replication off of therapy [40], other experience has been less favorable [37]. It is now clear that in most cases, simply starting therapy early is not sufficient to control viremia off treatment. Therefore, consideration has been given to novel strategies to enhance specific and nonspecific immunity. One example of this was to interrupt therapy in order to allow for transient viral rebound to enhance immune responses [33]. Although initial observations in a group of eight patients studied in Boston were favorable, further follow-up of a larger group has not shown sustained viral control [33,41]. Nevertheless, immunologic data still suggest that there may be unique benefits associated with early therapy and that further study is warranted. However, even if early treatment does prove to be clinically relevant, further research is needed to define how late is too late to derive such benefits, and how long treatment must be continued.

Other novel strategies being considered in order to enhance viral control in those treated during primary HIV-1 infection are the use of immunomodulators such as cyclosporine A and interleukin 2. One pilot study showed improved $\mathrm{CD}_{4}{ }^{+} \mathrm{T}$-lymphocyte numbers and enhanced immunologic function in those treated with cyclosporine A and antiretroviral therapy, compared with those administered antiretrovirals alone [42]. A larger randomized controlled trial has just recently been initiated to determine whether those treated with antiretrovirals and cyclosporine A will have enhanced virologic control upon treatment discontinuation, compared with those administered antiviral alone. In a recently reported study, concurrent treatment of primary infection with antiretrovirals and interleukin 2 resulted in increased $\mathrm{CD}^{+}{ }^{+} \mathrm{T}$-cell counts and early increases in CD8 ${ }^{+} \mathrm{T}$-cell noncytotoxic anti-HIV-1 responses, the clinical relevance of which is unknown [43]. The use of an HIV-1 immunogen also remains an area of great interest. Although the optimal reagents are not yet available, there are limited data showing enhancement of immune responses in those treated with antiretrovirals and an immunogen, albeit without clear virologic benefits [44].

Considering the limited data regarding the benefits of treatment during primary HIV-1 infection, it remains difficult to counsel those identified during this stage of disease. The most recent guidelines from the US Department of Health and Human Services suggest that patients be informed of the known risks and benefits of treatment during primary infection, without making any strong recommendations for or against therapy [22]. Treatment guidelines from the International AIDS Society-USA in 2000 suggested that treatment be discussed and offered to those with primary infection. In 2002, the same group mentioned treatment during this stage of disease only in the context of the potential rationale for treatment interruptions. In contrast, the most recent 2004 guidelines do not even mention a potential role for antiretroviral treatment during primary infection [45]. Other guideline committees have also weighed in on this issue. The British HIV Association guidelines make no strong recommendations regarding treatment, although they do state that therapy can be considered, particularly in the context of clinical trials [46]. Similarly, the most recent European Guidelines say that treatment should be considered for those identified with primary infection who are able to commit to therapy, particularly in the context of clinical trials [47].

In keeping with the ambivalence of these guidelines, the enthusiasm for treatment in practice appears to have changed with time. The French PRIMO multicenter cohort recently reported its experience with 291 patients identified with primary infection between 1996 and 2001 [48]. In 1996, 92\% of those with primary infection were treated, achieving undetectable levels of HIV-1 RNA ( $<50$ copies $/ \mathrm{mL}$ ) in $53 \%$, with adverse events occurring in just over $50 \%$ of patients. In contrast, by 2001 only $56 \%$ received treatment. Consequently, at this time it is reasonable to share with the patient the risks and potential benefits of early treatment and only consider initiating therapy in those who completely understand the issues and are committed to taking the treatment for some undefined period of time while awaiting further guidance from clinical research. Whenever possible, these patients should be encouraged to participate in clinical research designed to enhance our understanding of this unique stage of disease. 
In light of the prevalence of drug-resistant virus in treatment-naïve patients, it is certainly reasonable to perform baseline resistance testing at the time primary HIV-1 infection is diagnosed, regardless of whether one is intending to start therapy or not [21]. If the person chooses to opt for early therapy, it is unknown whether therapy should be withheld pending the results of drug resistance testing. Although some have proposed that pending the availability of resistance data the risk of developing additional mutations might be reduced by using a ritonavir-boosted protease inhibitor-based regimen, this strategy has not been tested prospectively. Assuming transmitted drug resistance is not an issue, there is reasonably good experience treating primary HIV1 infection with protease inhibitor- and non-nucleoside analogue reverse transcriptase inhibitor-based combination therapy $[49,50]$. The most important factors, as is the case in those who are chronically infected, are the willingness of the patient to commit to therapy, the patient's personal preferences for dosing schedule, and the overall tolerability of the regimen.

\section{Conclusions}

Primary HIV-1 infection represents the earliest stage of disease and is of great interest to the behavioral, clinical, and basic science communities. The diagnosis of primary infection requires that clinicians be aware of the signs, symptoms, and laboratory manifestations of this stage of disease, and understand the diagnostic tests needed to identify those in the seronegative window of infection. Identification of patients with primary infection allows for early counseling to prevent spread of infection to others and facilitates entry into medical care. It also allows newly infected individuals to be informed of the potential role that early antiretroviral therapy may have in preserving important HIV-1-specific immune responses, even if definitive recommendations regarding therapy can not yet be made. Regardless of whether these individuals choose to initiate therapy, they should be encouraged to participate in clinical research designed to advance our understanding of this highly dynamic stage of disease.

\section{Acknowledgments}

This work was funded with support from AI43638, HD41224, AI27660, and RR00425 from the National Institutes of Health, and CCTG-CC99 SD003 from the Universitywide AIDS Research Program.

\section{References and Recommended Reading}

Papers of particular interest, published recently,

have been highlighted as:

- Of importance

- Of major importance

1. Vanhems P, Dassa C, Lambert J, et al.: Comprehensive classification of symptoms and signs reported among 218 patients with acute HIV-1 infection. J Acquir Immune Defic Syndr 1999, 21:99-106.

2. Daar ES, Little SJ, Pitt J, et al.: Diagnosing primary HIV infection. Ann Intern Med 2001, 134:25-29.

3. Dorrucci M, Rezza G, Vlahov D, et al.: Clinical characteristics and prognostic value of acute retroviral syndrome among injecting drug users. AIDS 1995, 9:597-604.

4. Hecht FM, Busch MP, Rawal B, et al.: Use of laboratory tests and clinical symptoms for identification of primary HIV infection. AIDS 2002, 24:1119-1129.

5. Karon JM, Fleming PL, Steketee RW, De Cock KM: HIV in the United States at the turn of the century: an epidemic in transition. Am J Public Health 2001, 91:1060-1068.

6. Rottingen JA, Cameron DW, Garnett GP: A systematic review of the epidemiologic interactions between classic sexually transmitted diseases and HIV: how much really is known? Sex Trans Dis 2001, 28:579-597.

7. Richters J, Grulich A, Ellard J, et al.: HIV transmission among gay men through oral sex and other uncommon routes: case series of HIV seroconverters, Sydney. AIDS 2003, 17:2269-2271.

8. Xiridou M, Geskus R, de Wit J, et al.: Primary HIV infection as source of HIV transmission within steady and casual partnerships among homosexual men. AIDS 2004, 18:1311-1320.

This study provides modeled data that suggest that primary infection plays a substantial role in transmission in those with casual relationships. These data emphasize the importance of identifying those with primary infection to prevent further spread of HIV.

9. Weintrob AC, Giner J, Menezes P, et al.: Infrequent diagnosis of primary human immunodeficiency virus infection: missed opportunities in acute care settings. Arch Intern Med 2003, 163:2097-2100.

$10 . \bullet$ Fiebig E, Wright D, Rawal B, et al.: Dynamics of HIV viremia and antibody seroconversion in plasma donors: implications for diagnosis and staging of primary HIV infection. AIDS 2003, 17:1871-1879.

This represents one of the premiere studies that demonstrates the relationship between time since infection and the presence of various virologic and immunologic markers of HIV-1 infection.

11. Pilcher CD, McPherson JT, Leone PA, et al:: Real-time, universal screening for acute HIV infection in a routine HIV counseling and testing population. JAMA 2002, 288:216-221.

12. Burchell AN, Calzavara L, Ramuscak N, et al.: Symptomatic primary HIV infection or risk experiences? Circumstances surrounding HIV testing and diagnosis among recent seroconverters. Int J STD AIDS 2004, 14:601-608.

13. Constantine NT, Sill AM, Jack N, et al.: Improved classification of recent HIV-1 infection by employing a two-stage sensitive/less-sensitive test strategy. J Acquir Immune Defic Syndr 2003, 32:94-103.

14. Geijtenbeek TB, van Kooyk Y: DC-SIGN: a novel HIV receptor on DCs that mediates HIV-1 transmission. Curr Top Microbiol Immunol 2003, 276:31-54.

15. Delwart E, Magierowska M, Royz M, et al.: Homogeneous quasispecies in 16 out of 17 individuals during very early HIV-1 primary infection. AIDS 2002, 16:189-195.

16. Sagar M, Lavreys L, Baeten JM, et al.: Infection with multiple human immunodeficiency virus type 1 variants is associated with faster disease progression. J Virol 2003, 77:12921-12926. 
17. Weinstock HS, Zaidi I, Heneine W, et al.: The epidemiology of antiretroviral drug resistance among drug-naive HIV-1-infected persons in 10 US cities. J Infect Dis 2004, 189:2174-2180.

18. Blower SM, Aschenbach AN, Kahn JO: Predicting the transmission of drug-resistant HIV: comparing theory with data. Lancet Infect Dis 2003, 3:10-11.

19. Little SJ, Holte S, Routy JP, et al.: Antiretroviral-drug resistance among patients recently infected with HIV. N Engl J Med 2002, 347:385-394.

20. Barbour JD, Hecht FM, Wrin T, et al.: Persistence of primary drug resistance among recently HIV-1 infected adults. AIDS 2004, 18:1683-1689.

21. Hirsch MS, Brun-Vezinet F, Clotet B, et al.: Antiretroviral drug resistance testing in adults infected with human immunodeficiency virus type 1: 2003 recommendations of an International AIDS Society-USA Panel. Clin Infect Dis 2003, 37:113-128.

22. US Department of Health and Human Services Panel of Clinical Practices for Treatment of HIV Infection: Guidelines for the use of antiretroviral agents in HIV-1-infected adults and adolescents. http://www.aidsinfo.nih.gor. Accessed October 29, 2004.

23. Koup RA, Safrit JT, Cao Y, et al.: Temporal association of cellular immune responses with the initial control of viremia in primary human immunodeficiency virus type 1 syndrome. J Virol 1994, 68:4650-4655.

24. Musey L, Hughes J, Schacker T, et al.: Cytotoxic-T-cell responses, viral load, and disease progression in early human immunodeficiency virus type 1 infection. N Engl J Med 1997, 337:1267-1274.

25. Oxenius A, Price DA, Trkola A, et al.: Loss of viral control in early HIV-1 infection is temporally associated with sequential escape from CD8 + T cell responses and decrease in HIV-1-specific CD4+ and CD8+ T cell frequencies. J Infect Dis 2004, 190:713-721.

26. Richman DD, Wrin T, Little SJ, Petropoulos CJ: Rapid evolution of the neutralizing antibody response to HIV type 1 infection. Proc Natl Acad Sci U S A 2003, 100:4144-4149.

27. Montefiori DC, Altfeld M, Lee PK, et al:: Viremia control despite escape from a rapid and potent autologous neutralizing antibody response after therapy cessation in an HIV-1-infected individual. J Immunol 2003, 170:3906-3914.

28. Forthal DN, Landucci G, Daar ES: Antibody from patients with acute human immunodeficiency virus (HIV) infection inhibits primary strains of HIV type 1 in the presence of natural-killer effector cells. J Virol 2001, 75:6953-6961.

29. Abdelwahab SF, Cocchi F, Bagley KC, et al.: HIV-1suppressive factors are secreted by CD4+ T cells during primary immune responses. Proc Natl Acad Sci U S A 2003, 100:15006-15010.

30. Blattner WA, Ann Oursler K, Cleghorn F, et al.: Rapid clearance of virus after acute HIV-1 infection: correlates of risk of AIDS. J Infect Dis 2004, 189:1793-1801.

31. Rosenberg ES, Billingsley JM, Caliendo AM, et al.: Vigorous HIV-1-specific CD4+ T cell responses associated with control of viremia. Science 1997, 278:1447-1450.

32. Gloster SE, Newton P, Cornforth D, et al.: Association of strong virus-specific $\mathrm{CD} 4 \mathrm{~T}$ cell responses with efficient natural control of primary HIV-1 infection. AIDS 2004, 18:749-755.

33. Rosenberg ES, Altfeld M, Poon SH, et al.: Immune control of HIV-1 after early treatment of acute infection. Nature 2000, 407:523-526.
34. Kinloch-De Loes S, Hirschel BJ, Hoen B, et al: : A controlled trial of zidovudine in primary human immunodeficiency virus infection. N Engl J Med 1995, 333:408-413.

$35 . \bullet$ Smith D, Walker B, Cooper D, et al.: Is antiretroviral treatment of primary HIV infection clinically justified on the basis of current evidence? AIDS 2004, 18:709-718.

This is a comprehensive overview of the rationale and experience in treating those identified with primary HIV-1 infection.

36. Miller J, Carr A, Smith DK, et al.: Lipodystrophy following antiretroviral therapy of primary HIV infection. AIDS 2000, 14:2406-2407.

37. Daar ES, Bai J, Hausner MA, et al.: Acute syndrome following discontinuation of antiretroviral therapy in a patient treated prior to seroconversion. Ann Intern Med 1998, 128:827-829.

38. Tremblay CL, Hicks JL, Sutton L, et al.: Antiretroviral resistance associated with supervised treatment interruptions in treated acute HIV infection. AIDS 2003, 17:1086-1089.

39. Crepaz N, Hart TA, Marks G: Highly active antiretroviral therapy and sexual risk behavior: A meta-analytic review. JAMA 2004, 292:224-236.

40. Lisziewicz J, Rosenberg E, Lieberman J, et al.: Control of HIV despite the discontinuation of antiretroviral therapy. N Engl J Med 1999, 340:1683-1684.

41. Kaufmann DE, Lichterfeld M, Altfeld M, et al.: Limited durability of viral control following treated acute HIV infection. Plos Med 2004, 1:137-147.

42. Rizzardi GP, Harari A, Capiluppi B, et al.: Treatment of primary HIV-1 infection with cyclosporin A coupled with highly active antiretroviral therapy. J Clin Invest 2002, 109:681-688.

43. Martinez-Marino B, Ashlock BM, Shiboski S, et al.: Effect of IL-2 therapy on CD8+ cell noncytotoxic anti-HIV response during primary HIV-1 infection. J Clin Immunol 2004, 24:135-144.

44. Markowitz M, Jin X, Hurley A, et al:: Discontinuation of antiretroviral therapy commenced early during the course of human immunodeficiency virus type 1 infection, with or without adjunctive vaccination. J Infect Dis 2002, 186:634-643.

45. Yeni PG, Hammer SM, Hirsch MS, et al.: Treatment for adult HIV infection: 2004 recommendations of the International AIDS Society-USA Panel. JAMA 2004, 292:251-265.

46. British HIV Association guidelines for the treatment of HIV disease with antiretroviral therapy. http://www.bhiva.org guidelines/2003/hiv/index.htm. Accessed October 29, 2004

47. European guidelines for the clinical management and treatment of HIV-infected adults in Europe. AIDS 2003, $17: S 3-S 26$.

48. Schiffer V, Deveau C, Meyer L, et al.: Recent changes in the management of primary HIV infection: results from the French PRIMO Cohort. HIV Med 2004, 5:326-333.

49. Smith D, Berrey MM, Robertson M, et al.: Virological and immunological effects of combination antiretroviral therapy with zidovudine, lamivudine, and indinavir during primary human immunodeficiency virus type 1 infection. J Infect Dis 2000, 182:950-954.

50. Portsmouth S, Imami N, Pires A, et al.: Treatment of primary HIV-1 infection with nonnucleoside reverse transcriptase inhibitor-based therapy is effective and well tolerated. HIV Med 2004, 5:26-29. 